\title{
A Two Stage Op-Amp Phase Margin Symbolic Expression
}

\author{
Alexandru Gabriel Gheorghe ${ }^{1, *}$ and Mihai Eugen Marin ${ }^{1}$ \\ ${ }^{1}$ Department of Electrical Engineering, "Politehnica" University, Bucharest, Romania
}

\begin{abstract}
A phase margin symbolic expression of a two stage Miller compensated operational amplifier is computed in this paper. Using this expression, an analysis to evaluate the influence of the Miller and load capacitance on phase margin is performed. This way, a designer can rapidly choose the optimal set of values to fulfil an imposed phase margin. The phase margin expression is based on poles/zeros symbolic expressions obtained using a symbolic LR algorithm able to compute both the numerical values and the approximate symbolic expressions of poles and zeros of a circuit. The numerical values obtained with this algorithm are compared with those computed by SPECTRE. The example is a two stage Miller compensated operational amplifier designed in a 180nm CMOS technology.
\end{abstract}

\section{INTRODUCTION}

The symbolic expressions of poles and zeros are very useful in designing of analog linear circuit such as filters and amplifiers. These expressions can be used in solving stability problems or in circuit parameter identification. An example of this problem is the compensation capacitor calculation to assure the closed loop stability of a two stage operational amplifier for different capacitive loads. In the basic Miller compensation technique [1], a capacitance is connected across the two amplifier stages. The classical design approach is based on a simplified model having a frequency response with two left half plane poles and one right half plane zero for the amplifier in open loop configuration [2-4]. The schematic of a typical two stage operational amplifier Miller compensated designed in a $180 \mathrm{~nm}$ CMOS technology is presented in Fig. 1. The compensation network is constituted of the CM capacitance connected between the two amplifier stages. Some papers shows that actually the open loop operational amplifier transfer function is characterized by three poles and one zero [57]. Numerical pole/zero computation with SPECTRE, HSPICE [8] exhibit that the number of poles/zeros is larger. In this paper we compute the numerical values and the simplified symbolic expressions of all four poles and three zeros of an open loop operational amplifier. In Section 2 the algorithm used to compute the poles/zeros numerical and symbolic expressions, based on LR iterations and some simplifications is described [9, 10]. Section 3 contains an example, the poles/zeros numerical values obtained with our program compared with those given by SPECTRE, and the simplified symbolic expressions. In Section 4 the symbolic transfer function and symbolic phase margin expression is computed and is used to investigate the validity range for the compensation and the load capacitance set of a two stage operational amplifier Miller compensated used with a capacitive load. In Section 5 some conclusions are presented.

\section{IMPROVED ALGORITHM}

Consider a linear circuit for which the state equations in the normal form and the output equations are:

$$
\begin{aligned}
d x / d t & =A x+B u+E d u / d t \\
y & =C x+d u
\end{aligned}
$$

where $A$ is the state matrix, $x$ is the state variable vector, $u$ is the input vector having only one component, $y$ is the output vector having only one component, $d$ is a scalar, and the matrices $B, E, C$ have appropriate dimensions. The entries of all these matrices are ratios of polynomials in terms of the circuit parameters.

The poles numerical values are the eigenvalues of $A$, and the zeros numerical values are the eigenvalues of the "state-like" matrix $A$ ' determined with the inverse system method:

$$
A^{\prime}=\left(A-\frac{B C}{d}\right)\left(1+\frac{E C}{d}\right)^{-1}
$$

For a circuit with no excess state variables $E=0$ and no matrix inverse must be computed. The $A$ matrix can be computed by using the algorithm for the calculation of the symbolic hybrid matrix of a resistive multiport [8]. In case that $d=0$ the "state-like" matrix cannot be computed using (eq. 3) and Sandberg and So algorithms [11] or Davison algorithm [12] must be used.

The approximate symbolic expression of a pole or zero is computed as the eigenvalue of the state matrix or of the "state-like" matrix [10] using the following steps. The symbolic state and the "state-like" matrix whose entries contain the circuit parameters is obtained starting from a similar to SPICE input text file [10]. The circuit equations are simplified by replacing circuit elements

Corresponding author: alexandru.gheorghe@upb.ro 
with open-circuit and/or short-circuit in the increasing order of their relative differential sensitivities. An attempt is validated if the error introduced by a simplification is smaller than a user defined one. After that, attempts to cut an as large as possible group of the same index rows and columns are made, the remaining matrix having the imposed pole/zero as an eigenvalue. A reduced order symbolic state matrix is generated after each successful attempt. If this matrix has the order greater than two, the symbolic $L R$ iterations are performed using the matrix simplified in the previous steps. The user can set the simplification errors during each simplification stage. If this matrix has the order 1 or 2, the pole/zero expression can be found straightforwardly. The errors of the pole/zero expressions are computed in the design point and in the corners corresponding to all combinations of the extreme values. The procedures that implement this algorithm have been written in MAPLE.

\section{EXAMPLE}

Figure 1 a) presents a two stage operational amplifier with Miller compensation, designed in $180 \mathrm{~nm}$ technology and Fig. $1 \mathrm{~b}$ ) presents the test-bench circuit. Using DC operating point analysis from SPECTRE, the small signal parameters of the transistors have been extracted (Fig. 2).

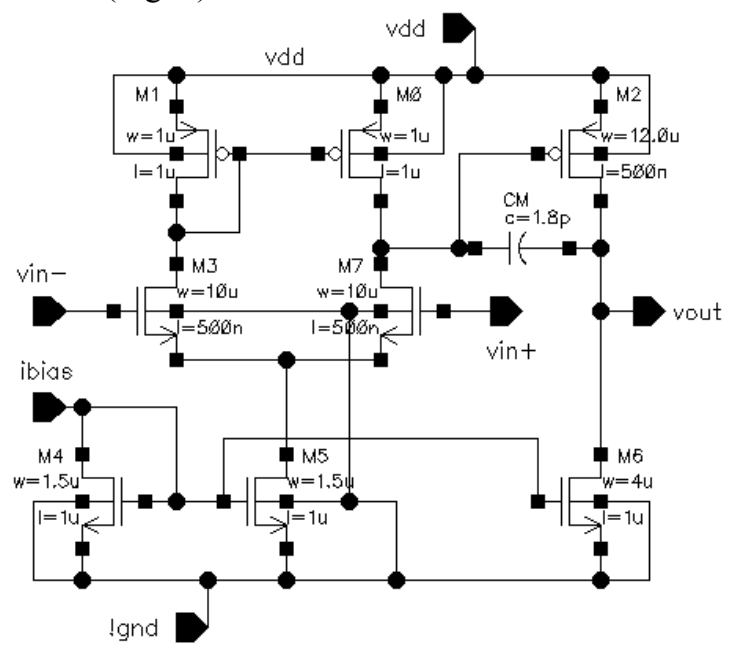

Fig. 1 a) A two stage operational amplifier with Miller compensation

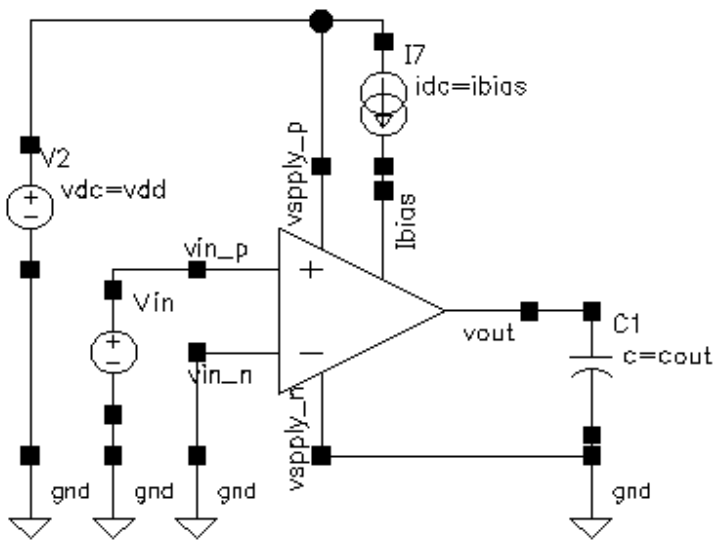

Fig. 1 b) The test-bench circuit
Two CMOS equivalent circuit models have been tested (Fig $2 \mathrm{a}, \mathrm{b}$ ) in order to obtain the same frequency and time response with a simple circuit. The results obtained with this two models (AC analysis in SPECTRE) are almost the same (Fig 3).

The operational amplifier in Fig. 1 has been simulated with SPECTRE and the algorithm presented in Section 2. In this picture in red is the response (magnitude in $\mathrm{dB}$ and phase in degree of the output voltage) of the operational amplifier using the first CMOS model and in green is the response using the second model. It can be observed that for a reasonable frequency band the two models have the same behavior. For further investigations the second CMOS model will be used.

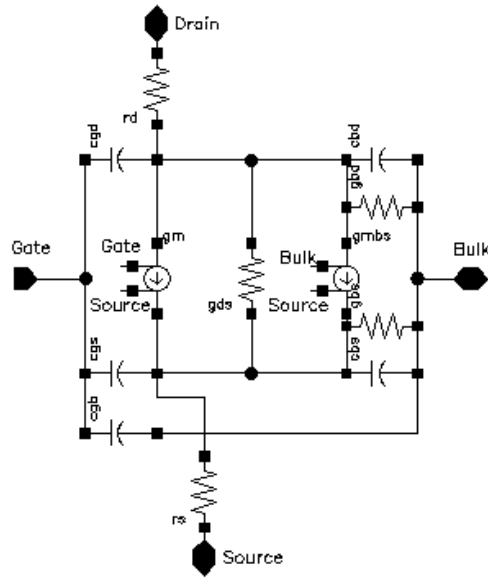

a) First model

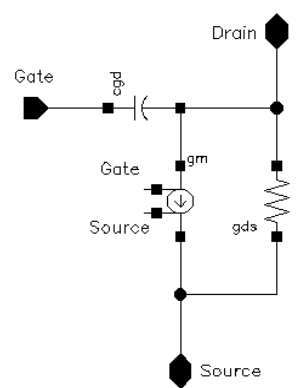

b) Second model
Fig. 2 The CMOS equivalent circuits

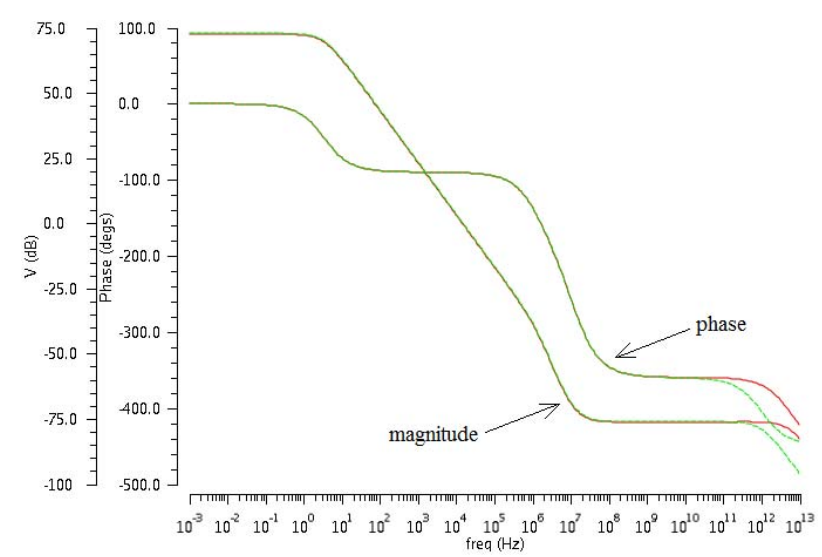

Fig. 3 The Op-Amp frequency response computed with SPECTRE

The numerical results for poles/zeros obtained with these programs are presented in the Table 1 and Table 2.

Table 1. Poles values

\begin{tabular}{|c|c|c|}
\hline & Proposed algorithm & SPECTRE \\
\hline p1 & $-3.68 \mathrm{E}+00$ & $-3.43 \mathrm{E}+00$ \\
\hline p2 & $-1.31 \mathrm{E}+06$ & $-1.39 \mathrm{E}+06$ \\
\hline p3 & $-2.32 \mathrm{E}+07$ & $-2.32 \mathrm{E}+07$ \\
\hline p4 & $-1.02 \mathrm{E}+12$ & - \\
\hline
\end{tabular}


Table 2. Zeros values

\begin{tabular}{|c|c|c|}
\hline & Proposed algorithm & SPECTRE \\
\hline z1 & $7.86 \mathrm{E}+06$ & $8.65 \mathrm{E}+06$ \\
\hline z2 & $1.50 \mathrm{E}+07$ & $1.50 \mathrm{E}+07$ \\
\hline z3 & $-2.38 \mathrm{E}+07$ & - \\
\hline
\end{tabular}

Even though the frequency characteristic computed by SPECTRE and the proposed algorithm (Fig 3 and Fig 4) exhibit a pole in the range of $1 \mathrm{E}+12 \mathrm{~Hz}$, the $\mathrm{PZ}$ analysis from SPECTRE cannot compute it even though the accuracy is increased.

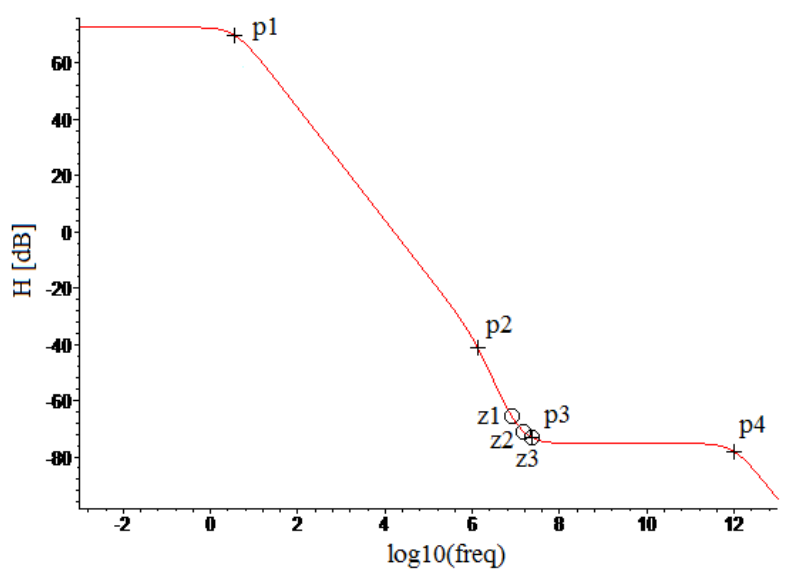

Fig. 4 The Op-Amp frequency response obtained with the proposed algorithm ("+" marks a pole and "॰" marks a zero)

The approximate symbolic expressions of poles obtained with the algorithm presented above are given in Table 3:

Table 3. Approximate symbolic pole expressions

\begin{tabular}{|c|c|c|}
\hline pole & Simplified expression & Error [\%] \\
\hline p1 & $-\frac{6.8710^{-15} C_{M}+1.2610^{-26}}{1.8510^{-12} C_{M} g_{m 2}+C_{M}^{2} g_{m 2}}$ & 3.06 \\
\hline p2 & $-\frac{g_{m 2}-5.2410^{-6}}{C_{o u t}}$ & 0.36 \\
\hline p3 & $-\frac{1.00}{\left(r_{d s 1}|| \frac{1}{g_{m 1}}\right) c_{g d}}$ & \\
\hline p4 & $-\frac{1.02}{r_{V i n} c_{g d 7}}$ & 0.86 \\
\hline
\end{tabular}

In Table 4 are given the approximate symbolic expressions of zeros obtained with the algorithm presented above.

In these expressions only two symbols, the ones with the greatest differential sensitivities are kept, the others being substituted with their numerical values. Having these expressions is interesting to see what part of the circuit give each of the pole/zero.

All poles and zeros simplified expressions are compared with the eigenvalues computed numerically for a range $\pm 30 \%$ of the nominal parameter value. There is a good agreement between these approximate formulas and the numerical values, the maximum error in the nominal point being less than $4 \%$.

Table 4. Approximate symbolic zero expressions

\begin{tabular}{|c|c|c|}
\hline zero & Simplified expression & $\begin{array}{c}\text { Error } \\
{[\%]}\end{array}$ \\
\hline z1 & $\frac{6.4410^{-16} g_{m 7}^{2}+4.1110^{-8} c_{g d 7} g_{m 7}}{4.1810^{-8} c_{g d 7}^{2}+7.6310^{-16} c_{g d 7} g_{m 7}}$ & 0.95 \\
\hline z2 & $\frac{g_{m 2}}{C_{M}}$ & 0.32 \\
\hline z3 & $-\frac{1.00}{\left(r_{d s 1}|| \frac{1}{g_{m 1}}\right) c_{g d 0}}$ & 3.68 \\
\hline
\end{tabular}

\section{THE SYMBOLIC PHASE MARGIN}

Starting from the simplified $\mathrm{p} / \mathrm{z}$ expressions, the transfer function is computed with the following formulae:

$$
H(s)=K \frac{\left(s+z_{1}\right)\left(s+z_{2}\right)\left(s+z_{3}\right)}{\left(s+p_{1}\right)\left(s+p_{2}\right)\left(s+p_{3}\right)\left(s+p_{4}\right)}
$$

Keeping as symbols only the Miller and the load capacitance it result:

$$
\begin{aligned}
& H(s)=\frac{3.310^{19} C_{\text {out }}\left(C_{M}+1.910^{-12}\right)\left(s C_{M}-1.710^{-4}\right)}{\left(1.610^{-13} s+1.0\right)\left(6.910^{-9} s+1\right)\left(s C_{\text {out }}+1.610^{-4}\right)} . \\
& \cdot \frac{\left(8.210^{-37} s-4.010^{-29}\right)\left(6.910^{-9} s-1.0\right)}{\left(1.710^{-4} s C_{M}^{2}+3.110^{-16} s C_{M}+6.910^{-15} C_{M}+1.310^{-26}\right)}
\end{aligned}
$$

The symbolic phase margin expression is calculated using the well known formulae [13]:

$$
P M=180^{\circ}+\operatorname{arctg}\left(\frac{\operatorname{Im}\left(H\left(j \omega_{g c}\right)\right)}{\operatorname{Re}\left(H\left(j \omega_{g c}\right)\right)}\right) .
$$

The $\omega_{g c}$ is the pulsation for unity magnitude, obtained solving:

$$
\left|H\left(j \omega_{g c}\right)\right|^{2}=1 \text { or }\left|N\left(j \omega_{g c}\right)\right|^{2}=\left|D\left(j \omega_{g c}\right)\right|^{2}
$$

Using the nominal parameters $\left(C_{\text {out }}=20 \mathrm{pF}, C_{M}=1.8 \mathrm{pF}\right)$, the values in Table 5 are obtained for phase margin. Having a symbolic expression is very useful to verify the influence of various circuit parameters on the phase margin. For example in Fig. 5 can be observed the phase margin variation with $C_{M}$ and $C_{\text {out }}$. The stability range for phase margin is between $45^{\circ}$ and $135^{\circ}$, and the blue dot is the value of phase margin in the nominal design point.

Table 5. Numerical phase margin

\begin{tabular}{|c|c|c|}
\hline & Proposed algorithm & SPECTRE \\
\hline PM & $89.04^{\circ}$ & $89.24^{\circ}$ \\
\hline
\end{tabular}

In Table 6, using the phase margin expression, some values are computed showing the validity range for $\left(C_{M}\right.$, $C_{\text {out }}$ ) set and the relative errors $\varepsilon_{\text {rel }}$ to numerical values obtained with the PZ analysis from SPECTRE. 


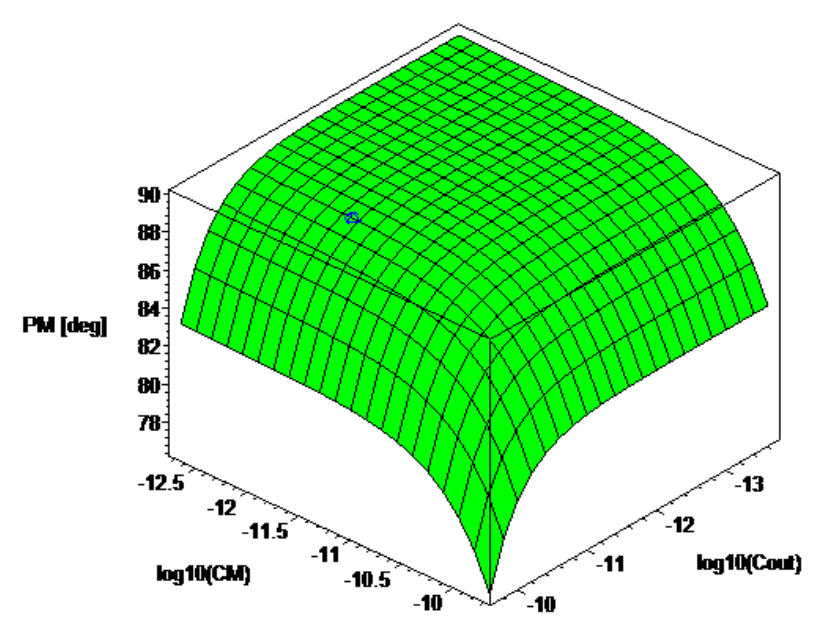

Fig. 5 Phase margin as a function of Miller and load capacitance

Table 6. Phase margin for various $C_{M}, C_{\text {out }}$ and the $\varepsilon_{r e l} \%$.

\begin{tabular}{|c|c|c|c|c|c|}
\hline \multirow{2}{*}{$0.2 \mathrm{pF}$} & $20 \mathrm{fF}$ & $0.2 \mathrm{pF}$ & $2 \mathrm{pF}$ & $20 \mathrm{pF}$ & $0.2 \mathrm{nF}$ \\
\cline { 2 - 6 } & 89.92 & 89.91 & 89.85 & 89.24 & 83.17 \\
\hline \multirow{2}{*}{$2 \mathrm{pF}$} & $0.97 \%$ & $1.02 \%$ & $1.50 \%$ & $6.47 \%$ & $37.76 \%$ \\
\cline { 2 - 6 } & 89.75 & 89.75 & 89.69 & 89.08 & 83.00 \\
\hline \multirow{2}{*}{$20 \mathrm{pF}$} & $0.13 \%$ & $0.13 \%$ & $0.14 \%$ & $0.26 \%$ & $1.66 \%$ \\
\hline \multirow{2}{*}{$0.2 \mathrm{nF}$} & 89.15 & 89.15 & 89.08 & 88.47 & 82.40 \\
\cline { 2 - 6 } & $0.90 \%$ & $0.90 \%$ & $0.96 \%$ & $1.60 \%$ & $8.48 \%$ \\
\cline { 2 - 6 } & $8.05 \%$ & $8.05 \%$ & $8.12 \%$ & $8.92 \%$ & $17.50 \%$ \\
\hline
\end{tabular}

\section{CONCLUSIONS}

A procedure implemented in MAPLE is used to compute both numeric and symbolic poles/zeros, and phase margin expressions. The numerical results are compared with those obtained using SPECTRE. In this case, even though the frequency characteristic computed by SPECTRE exhibit a pole in the range of $10^{12} \mathrm{~Hz}$, the PZ analysis from SPECTRE cannot compute it even though the accuracy is increased.

A symbolic expression of the phase margin is obtained function of Miller and the load capacitance for an operational amplifier designed in an 180nm technology. Varying both capacitances, the validity range of the optimum phase margin can be easily obtained. Of course the transfer function and the symbolic expression of the phase margin can be obtained as a function of other desired symbols.

Having the poles, zeros, and phase margin expressions, first is interesting to see what part of the circuit give each of the expression and second, gives designers the possibility to adjust the project changing only a small part of the circuit (e.g. a transistor, a capacitor or a resistor).

This work has been funded by University Politehnica of Bucharest, through the "Excellence Research Grants" Program,
UPB - GEX 2017. Identifier: UPB- GEX2017, Ctr. No.7/2017". Dr. A. G. Gheorghe thanks to professor F. Constantinescu, Department of Electrical Engineering, "Politehnica" University from Bucharest, for his help.

\section{References}

1. Z. Yan, P.I. Mak, R.P. Martins, "Two Stage Operational Amplifiers: Power and Area Efficient Frequency Compensation for Driving a Wide Range of Capacitive Load", IEEE Circuits and Systems Magazine, (2011).

2. H. J. Yang, and D. J. Allstot, "Considerations for Fast Settling Operational Amplifiers," IEEE Trans. On Circ. and Syst., vol. 37, no. 3, pp.326-34, (Mar. 1990).

3. U. Chilakapati and T. Fiez, "Effect of Switch Resistance on the SC Integrator Settling Time," IEEE Trans. On Circ. and Syst. II: Analog and Digital Sig. Proc., vol. 46, no. 6, pp. 810-815, (Jun. 1999).

4. U. Chilakapati and T. Fiez, "Settling time design considerations for SC integrators," IEEE Int. Symp. on Circ. And Syst., pp. 492-495, (Jun. 1998).

5. G. Palumbo and S. Pennisi, Feedback Amplifiers Theory and Design. Boston, MA: Kluwer, (2002).

6. G. Palmisano, G. Palumbo and S. Pennisi, "Design Procedure for Two-Stage CMOS Transconductance Operational Amplifiers: A Tutorial," Analog Integrated Circuits and Signal Processing, vol. 27, pp. 179-189, (2001).

7. F. A. Amoroso, A. Pugliese, G. Cappuccino, Design Considerations for Fast-Settling Two-Stage MillerCompensated Operational Amplifiers, Electronics, Circuits, and Systems, 2009. ICECS 2009, (2009), Page(s): $5-8$.

8. A. G. Gheorghe, F. Constantinescu, "Pole/zero computation for linear circuits", 6-th European Symposium on Computer Modeling and Simulation, Malta, (Nov. 14-16, 2012).

9. A. G. Gheorghe, F. Constantinescu, M. Nitescu, "Improved LR Algorithm for Computation of the Approximate Symbolic Pole/Zero Expressions", AFRICON 2013, Mauritius, (9-12 sept., 2013).

10. A. G. Gheorghe, F. Constantinescu, M. Niţescu , "State Matrix Simplification for Computation of the Approximate Pole/Zero Expressions", International Conference on Synthesis, Modeling, Analysis and Simulation Methods and Applications to Circuit Design (SMACD 2015), Istanbul, Turkey, (7-9 September 2015).

11. I. W. Sanberg, H. C. So, "A two-sets-of-eigenvalues approach to the computer analysis of linear systems", IEEE Transactions on Circuit Theory, vol. CT-16, No. 4 (November 1969), pp509-517.

12. E. J. Davison, "On the calculation of zeros of a linear constant system", IEEE Transactions on Circuit Theory, vol. CT-18, No. 1 (January 1971), pp. 183-184.

13. Behzad Razavi, Design of Analog CMOS Integrated Circuits, McGrawHill, (2001). 